PROCEEDINGS OF THE

AMERICAN MATHEMATICAL SOCIETY

Volume 132, Number 11, Pages 3397-3409

S 0002-9939(04)07346-0

Article electronically published on June 16, 2004

\title{
CELLULAR GENERATORS
}

\author{
WOJCIECH CHACHÓLSKI, PAUL-EUGENE PARENT, AND DONALD STANLEY
}

(Communicated by Paul Goerss)

\begin{abstract}
The aim of this paper is twofold. On the one hand, we show that the kernel $\overline{C(A)}$ of the Bousfield periodization functor $P_{A}$ is cellularly generated by a space $B$, i.e., we construct a space $B$ such that the smallest closed class $C(B)$ containing $B$ is exactly $\overline{C(A)}$. On the other hand, we show that the partial order (Spaces, $\gg$ ) is a complete lattice, where $B \gg A$ if $B \in C(A)$. Finally, as a corollary we obtain Bousfield's theorem, which states that $($ Spaces,$>)$ is a complete lattice, where $B>A$ if $B \in \overline{C(A)}$.
\end{abstract}

\section{INTRODUCTION}

The main objects of our investigation are classes of unpointed spaces $\mathcal{C}$ that are closed under certain fundamental operations. We say that:

a. $\mathcal{C}$ is closed under weak equivalences if $X \in \mathcal{C}$ weakly equivalent to $Y$ implies that $Y \in \mathcal{C}$.

b. $\mathcal{C}$ is closed under taking homotopy colimits indexed by contractible categories if, for any diagram $F: I \rightarrow$ Spaces where $F(i) \in \mathcal{C}, i \in I$, and $I$ is contractible, hocolim $_{I} F \in \mathcal{C}$.

c. $\mathcal{C}$ is closed under taking pointed homotopy colimits if, for any pointed diagram $F: I \rightarrow$ Spaces $_{*}$ where $F(i) \in \mathcal{C}, i \in I$, the pointed homotopy colimit hocolim ${ }_{I}^{*} F \in \mathcal{C}$.

d. $\mathcal{C}$ is closed under taking basic operations if the following conditions are satisfied: for any set $I$, if $X_{i} \in \mathcal{C}, i \in I$, then, for any choice of basepoints in $X_{i}, \bigvee_{I} X_{i} \in \mathcal{C}$; if $X_{i} \in \mathcal{C}$, for $i=0,1,2$, then $\operatorname{hocolim}\left(X_{0} \leftarrow X_{1} \rightarrow X_{2}\right) \in \mathcal{C}$.

e. We say that $\mathcal{C}$ is closed under extensions by fibration if, for any map $X \rightarrow B$ such that $B \in \mathcal{C}$ and over any basepoint in $B$ the homotopy fiber $F i b(X \rightarrow$ $B) \in \mathcal{C}$, the space $X \in \mathcal{C}$.

Our primary interest is in closed classes which were introduced by E. Dror Farjoun $[8,9]$.

1.1. Definition. A class of unpointed spaces is closed if it consists of nonempty spaces, it is closed under weak equivalences, and it is closed under taking homotopy colimits indexed by contractible categories.

Received by the editors November 1, 2000 and, in revised form, January 1, 2001.

2000 Mathematics Subject Classification. Primary 55Q05.

The first author was partially supported by the NSF grant DMS-9803766.

This work has been partly supported by the Volkswagenstiftung Oberwolfach.

(C)2004 American Mathematical Society 
1.2. Remark. Although we deal with unpointed spaces, closed classes can be characterized using pointed conditions. These conditions are usually easier to check. It is a nontrivial result of E. Dror Farjoun that $\mathcal{C}$ is closed if and only if it is closed under weak equivalences and taking pointed homotopy colimits (see [4]). It is then not difficult to show that $\mathcal{C}$ is closed if and only if it is closed under weak equivalences and basic operations (see [4]). Throughout the paper we will use these characterizations of closed classes interchangeably.

The motivation for studying closed classes comes from the theory of unstable localization and colocalization functors (see [2, 9]), which are related to our key examples:

1.3. Example (Dror Farjoun, [8]). Let $A$ be a nonempty space. Denote by $\mathcal{C}(A)$ the smallest closed class that contains $A$. The relation $X \in \mathcal{C}(A)$ is denoted by $X \gg A$ and $X$ is said to be $A$-cellular. This defines an order on the class of all the spaces. The class $\mathcal{C}(A)$ can be identified with the image of the composition Spaces $_{*} \stackrel{C W_{A}}{\longrightarrow}$ Spaces $_{*} \rightarrow$ Spaces of the Dror Farjoun functor $C W_{A}$ and the forgetful functor (see [8, 5]).

1.4. Example (Bousfield, Dror Farjoun, [1, 9]). Let $A$ be a nonempty space. Denote by $\overline{\mathcal{C}(A)}$ the smallest closed class that contains $A$ and is closed under extensions by fibrations. The relation $X \in \overline{\mathcal{C}(A)}$ is denoted by $X>A$, and $X$ is said to be $A$-acyclic. This defines an order on the class of all the spaces. The class $\overline{\mathcal{C}(A)}$ can be identified with the kernel of the Bousfield functor $P_{A}$ (see [5]).

In this paper we look at certain global features of the orders $($ Spaces, $\gg)$ and $($ Spaces, $>$ ). Our work was inspired by a result of Bousfield 2], which says that (Spaces, $>$ ) is a complete lattice. Recall that an ordered class $(\mathcal{C},>)$ is called a complete lattice if it satisfies the following condition. For any set $\mathcal{J} \subset \mathcal{C}$, there are objects $\sup (\mathcal{J})$ and $\inf (\mathcal{J})$ in $\mathcal{C}$, such that: if $X \in \mathcal{J}$, then $\sup (\mathcal{J})>X>\inf (\mathcal{J})$, and for any other objects $Y$ and $Z$ in $\mathcal{C}$ for which $Y>X>Z$, if $X \in \mathcal{J}$, we have $Y>\sup (\mathcal{J})$ and $\inf (\mathcal{J})>Z$.

The main result of our paper is:

\subsection{Theorem.}

(1) The order $($ Spaces, $\gg)$ is a complete lattice.

(2) any space $A$ there exists a space $B$ such that $\overline{\mathcal{C}(A)}=\mathcal{C}(B)$. If $A$ is finite, then we can take $B$ to be the wedge of representatives of the isomorphism classes of finite A-acyclic spaces.

The strategy to prove this theorem is to look for special kinds of generators of a given class $\mathcal{C}$. We say that a space $A$ is a cellular generator of $\mathcal{C}$ if $\mathcal{C}=\mathcal{C}(A)$. We say that a set $\mathcal{B} \subset \mathcal{C}$ strongly generates $\mathcal{C}$ if any space in $\mathcal{C}$ is weakly equivalent to the homotopy colimit of a diagram $F: I \rightarrow$ Spaces, where $I$ is contractible and $F(i) \in$ $\mathcal{B}$, for $i \in I$. Strong generation is related to an invariant called "complication", see 6]. Observe that if $\mathcal{B}$ strongly generates $\mathcal{C}$, then, for any choice of basepoints in the elements of $\mathcal{B}, \bigvee_{A \in \mathcal{B}} A$ is a cellular generator of $\mathcal{C}$.

The key step in the proof of Theorem 1.5 is a generalization of the following statement to an arbitrary space $A$ :

1.6. Theorem. If $A$ is finite, then a set of representatives of isomorphism classes of finite A-acyclic (cellular) spaces strongly generates the class of A-acyclic (cellular) spaces. 


\section{Notation}

This paper is written simplicially. The symbols Spaces and Spaces* denote the categories of unpointed and pointed simplicial sets, respectively. We refer to objects of these categories as spaces. All the spaces and maps, unless clearly specified, are assumed to be unpointed.

The $n$-dimensional simplex is denoted by $\Delta[n]$. Its boundary is denoted by $\partial \Delta[n]$. The symbol $\Delta[n, k]$ denotes the horn in $\Delta[n]$. It is a simplicial subset of $\partial \Delta[n]$ generated by all except the $k$-th face of $\Delta[n]$. We refer to [7, 10] for the details of how to do homotopy theory in Spaces and Spaces ${ }_{*}$. Symbols: $\hookrightarrow, \rightarrow$, and $\stackrel{\sim}{\rightarrow}$ are used to denote respectively a cofibration, a fibration, and a weak equivalence in Spaces.

Let $K$ be a space. The cardinality of its set of all the nondegenerate simplices is denoted by $\# K$.

By $\mathbf{N}$ we denote the natural order on the set of natural numbers $\{0,1, \ldots\}$.

Let $\lambda$ be an ordinal number. By $[\lambda]$ we denote a category whose objects are ordinals $\kappa$ such that $\kappa<\lambda$, and $\operatorname{mor}_{[\lambda]}(\kappa, \delta)$ is either empty, if $\kappa>\delta$, or consists of a single element, if $\kappa \leq \delta$.

Let $J$ be a set and $\lambda$ be a limit ordinal. We say that $\# J$ is smaller than the cofinality of $\lambda$ if the following condition is satisfied. If $F:[\lambda] \rightarrow$ Sets is a functor where, for $\kappa<\lambda$, $\operatorname{colim}_{[\kappa]} F \rightarrow F(\kappa)$ is a monomorphism, then the natural map $\operatorname{colim}_{[\lambda]} \operatorname{mor}_{\text {Sets }}(J, F) \rightarrow \operatorname{mor}_{\text {Sets }}\left(J, \operatorname{colim}_{[\lambda]} F\right)$ is an isomorphism. For example, the cardinality of a set is smaller than the cofinality of $\mathbf{N}$ if and only if this set is finite. We refer the reader to [6] for a detailed discussion of this condition.

The symbols hocolim $F$ and hocolim ${ }_{I}^{*} G$ denote respectively the homotopy colimit and the pointed homotopy colimit of $F: I \rightarrow$ Spaces and $G: I \rightarrow$ Spaces $_{*}$ as defined in [3].

Let $F: I \rightarrow$ Spaces be a functor. Assume that the cardinality of the set of objects of $I$ is smaller than the cofinality of $\lambda$. If for any $i \in I$, \#F(i) is also smaller than the cofinality of $\lambda$, then the same is true for \# $\#$ colim ${ }_{I} F$ and \#hocolim $F$.

A category is called filtered if, for any two objects $a$ and $b$, there are morphisms $a \rightarrow c$ and $b \rightarrow c$, and, for any two morphisms $\alpha: a \rightarrow b$ and $\beta: a \rightarrow c$, there are morphisms $\alpha^{\prime}: b \rightarrow d$ and $\beta^{\prime}: c \rightarrow d$ such that $\alpha^{\prime} \alpha=\beta^{\prime} \beta$. If $I$ is filtered, then $I$ is contractible.

\section{Approximations}

Let $\mathcal{B}$ be a set of spaces. To see that $\mathcal{B}$ strongly generates $\mathcal{C}$, we need a criterion which guarantees that a space can be expressed as the homotopy colimit of a diagram indexed by a contractible category with values in $\mathcal{B}$.

Let $X$ be a space. Define $\mathcal{B} \downarrow X$ to be a category whose objects are maps $Y \rightarrow X$ where $Y \in \mathcal{B}$, and a set of morphisms between $\alpha_{0}: Y_{0} \rightarrow X$ and $\alpha_{1}: Y_{1} \rightarrow X$ consists of cofibrations $i: Y_{0} \hookrightarrow Y_{1}$ such that $\alpha_{1} i=\alpha_{0}$. By $\omega_{\mathcal{B}}: \mathcal{B} \downarrow X \rightarrow$ Spaces let us denote the forgetful functor $(Y \rightarrow X) \mapsto Y$.

3.1. Proposition. Let $\mathcal{B}$ be a set of connected spaces. Assume:

- The category $\mathcal{B} \downarrow X$ is filtered.

- If $K$ is a finite complex, then any map $K \rightarrow X$ can be expressed as a composition $K \rightarrow Y \rightarrow X$ where $Y \in \mathcal{B}$. Moreover, any commutative diagram of the 
form:

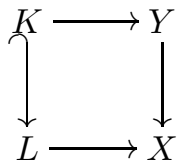

where $K, L$ are finite, $Y \in \mathcal{B}$, and $K \hookrightarrow L$ is a cofibration, can be extended to a commutative diagram:

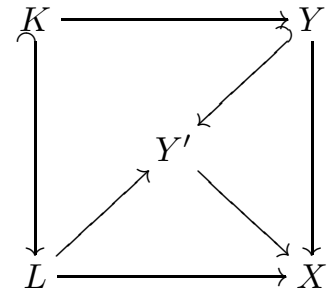

where $Y^{\prime} \in \mathcal{B}$ and $Y \hookrightarrow Y^{\prime}$ is a cofibration.

Then the map hocolim ${ }_{\mathcal{B} \downarrow X}\left(\omega_{\mathcal{B}}\right) \rightarrow X$ is a weak equivalence.

Proof. Let us denote the map hocolim ${ }_{\mathcal{B} \downarrow X}\left(\omega_{\mathcal{B}}\right) \rightarrow X$ by $f$. Since $\mathcal{B} \downarrow X$ is connected (it is even contractible) and since all the values of $\omega_{\mathcal{B}}$ are connected, then so is the

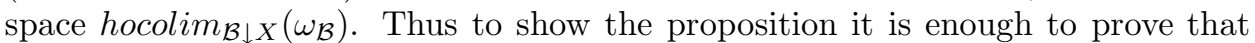
$f$ induces an isomorphism on homotopy groups $\pi_{*}$, for $* \geq 0$, for some choice of basepoints.

Observe that any pointed map $\alpha: K \rightarrow X$, where $K$ is finite, factors through $f$. To see this let $K \rightarrow Y \rightarrow X$ be a factorization of $\alpha$ given by the assumption. Let $Y \rightarrow$ hocolim $_{\mathcal{B} \downarrow X}\left(\omega_{\mathcal{B}}\right)$ be the map induced by $Y \rightarrow X$ in $\mathcal{B} \downarrow X$. It is clear that the composition $K \rightarrow Y \rightarrow$ hocolim ${ }_{\mathcal{B} \downarrow X}\left(\omega_{\mathcal{B}}\right) \rightarrow X$ equals $\alpha$.

An element in $\pi_{*} X$ is represented by a map $\alpha: s d^{k} \partial \Delta[n] \rightarrow X$ where $s d^{k} \partial \Delta[n]$ denotes the $k$-th barycentric subdivision of $\partial \Delta[n]$. Since $s d^{k} \partial \Delta[n]$ is finite, the above remark shows that $\alpha$ factors through $f$. It follows that $f$ induces an epimorphism on $\pi_{*}$. In particular, $X$ is connected.

Since $\mathcal{B} \downarrow X$ is filtered, any element of $\pi_{*} \operatorname{hocolim}_{\mathcal{B} \downarrow X}\left(\omega_{\mathcal{B}}\right)$ can be represented by a map $\alpha: s d^{k} \partial \Delta[n] \rightarrow Y$, for some $Y \rightarrow X$ in $\mathcal{B} \downarrow X$. Assume that $\alpha$ represents an element in the kernel of $\pi_{*} f$. This means that after possibly further subdividing there is a commutative diagram:

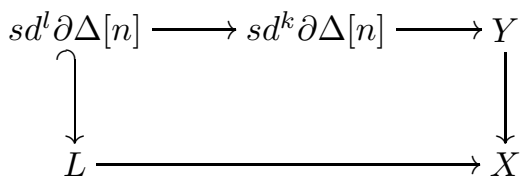

where $L$ is finite and contractible, for example the cone on $s d^{l} \partial \Delta[n]$. By the assumptions we can then find $Y^{\prime} \in \mathcal{B}$, for which the above diagram can be extended to:

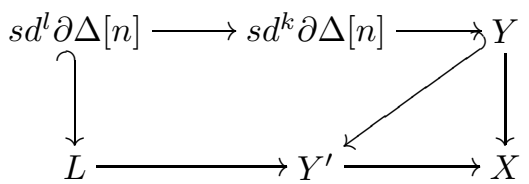

It follows that $\alpha$ represents a trivial class in the homotopy groups of hocolim $m_{\mathcal{B} \downarrow X}\left(\omega_{\mathcal{B}}\right)$ and thus $\pi_{*} f$ is also a monomorphism. 


\section{4. "SMALL" $\mathcal{C}$-SPACES}

In order to find strong generators of closed classes we are going to consider spaces for which a relative Quillen's small object argument holds. Let $\mathcal{C}$ be a class of spaces and $\lambda$ be a limit ordinal. Note that the collection of isomorphism classes of spaces in $\mathcal{C}$, whose cardinality is smaller than the cofinality of $\lambda$, forms a set. We denote this set by $\mathcal{C}_{\ll \lambda}$. Sometimes, by slight abuse of language, we say that $X$ belongs to $\mathcal{C}_{\ll \lambda}$ if $X$ is isomorphic to a space in $\mathcal{C}$ whose cardinality is smaller than the cofinality of $\lambda$. For example, $\mathcal{C}_{\ll \mathbf{N}}$ consists of finite complexes that belong to $\mathcal{C}$.

4.1. Definition. Let $\mathcal{C}$ be a class of spaces and $\lambda$ be a limit ordinal. A space $X$ is called $\lambda$-C-small if, for any map $K \rightarrow X$ such that \#K is smaller than the cofinality of $\lambda$, there exists a factorization $K \rightarrow Y \rightarrow X$ where $Y \in \mathcal{C}_{\ll \lambda}$.

In the case that $\mathcal{C}$ is closed under weak equivalences we can always arrange so that the map $K \rightarrow Y$, in the above definition, is a cofibration. To do that just take $Y^{\prime}=\operatorname{colim}(K \times \Delta[1] \hookleftarrow K \rightarrow Y)$.

The above definition is motivated by Proposition 3.1

4.2. Lemma. Let $\mathcal{C}$ be a closed class and $\lambda$ be a limit ordinal. Assume that $\mathcal{C}$ consists of connected spaces. If $X$ is $\lambda$-C-small, then the set $\mathcal{C}_{\ll \lambda}$ satisfies the assumptions of Proposition 3.1.

Proof. Since $\mathcal{C}$ consists of connected spaces, any $\lambda$-C $\mathcal{C}$-small space is connected. Let $\alpha: A \rightarrow X$ and $\beta: B \rightarrow X$ be elements of $\mathcal{C}_{\ll \lambda} \downarrow X$. Since $X$ is connected, there is a finite contractible space $L$ and a commutative diagram:

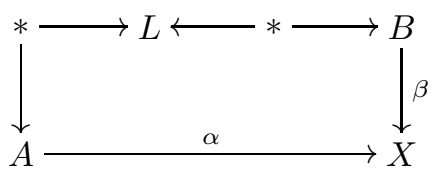

The map $\operatorname{hocolim}(A \leftarrow * \rightarrow L \leftarrow * \rightarrow B) \rightarrow X$ belongs to $\mathcal{C}_{\ll \lambda} \downarrow X$. Moreover, since $\mathcal{C}$ is closed, for any morphisms $A \hookrightarrow B$ and $A \hookrightarrow C$ in $\mathcal{C}_{\ll \lambda} \downarrow X$, the space $D=\operatorname{colim}(B \hookleftarrow A \hookrightarrow C)$ belongs to $\mathcal{C}_{\ll \lambda}$. This shows that $\mathcal{C}_{\ll \lambda} \downarrow X$ is filtered.

Consider now a commutative diagram:

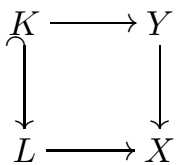

where $K, L$ are finite, $Y \in \mathcal{C}_{\ll \lambda}$, and $K \hookrightarrow L$ is a cofibration.

The push-out $Z=\operatorname{colim}(L \hookleftarrow K \rightarrow Y)$ clearly has cardinality smaller than the cofinality of $\lambda$. Since $X$ is $\lambda$-C-small, the induced map $Z \rightarrow X$ can be factored as $Z \hookrightarrow Y^{\prime} \rightarrow X$, where $Y^{\prime} \in \mathcal{C}_{\ll \lambda}$. This shows that the second condition of Proposition 3.1 is also satisfied.

Being $\lambda$-C - -small is not a homotopy invariant condition. To make it so we need a modification:

4.3. Proposition. Let $\mathcal{C}$ be a closed class and $\lambda$ be a limit ordinal whose cofinality is bigger than $\mathbf{N}$ or $\lambda=\mathbf{N}$. Then the following is a closed class:

$$
\mathcal{C}[\lambda]=\left\{X \mid \text { there exists } X \stackrel{\sim}{\rightarrow} X^{\prime} \text { such that } X^{\prime} \text { is } \lambda \text {-C } \mathcal{C} \text {-small }\right\} \text {. }
$$


4.4. Proposition. Let $\lambda$ be a limit ordinal whose cofinality is bigger than $\mathbf{N}$. If $\left\{\mathcal{C}_{i}\right\}_{i \in I}$ is a family of closed classes such that \#I is smaller than the cofinality of $\lambda$, then:

$$
\left(\bigcap_{i \in I} \mathcal{C}_{i}\right)[\lambda]=\bigcap_{i \in I}\left(\mathcal{C}_{i}[\lambda]\right)
$$

Propositions 4.3 and 4.4 will be proved at the end of the section.

4.5. Corollary. Let $\mathcal{C}$ be a closed class and $\lambda$ be a limit ordinal whose cofinality is bigger than $\mathbf{N}$ or $\lambda=\mathbf{N}$. Then $\mathcal{C}_{\ll \lambda}$ strongly generates $\mathcal{C}[\lambda]$ and, for any choice of basepoints in the elements of $\mathcal{C}_{\ll \lambda}, \mathcal{C}[\lambda]=\mathcal{C}\left(\bigvee_{A \in \mathcal{C}_{\ll \lambda}} A\right)$.

Proof. It is clear that $\mathcal{C}_{\ll \lambda} \subset \mathcal{C}[\lambda]$.

If $\mathcal{C}$ contains a non-connected space, then $\mathcal{C}$ consists of all the spaces (since $\mathcal{C}$ is closed). In particular, all discrete spaces belong to $\mathcal{C}$. In this case the corollary is clear since any $\lambda$-C $\mathcal{C}$-small space can be expressed as the realization (the homotopy colimit) of a simplicial space whose values are discrete spaces of cardinality smaller than the cofinality of $\lambda$.

If $\mathcal{C}$ consists of connected spaces, the corollary follows from Lemma 4.2

We can now prove the "cellular" part of Theorem 1.6

4.6. Corollary. Let $A$ be a space. Assume that either $\lambda=\mathbf{N}$, if $A$ is finite, or $\lambda$ is a limit ordinal whose cofinality is bigger than $\# A$, if \#A is infinite. Then $\mathcal{C}(A)=\mathcal{C}(A)[\lambda]$ and $\mathcal{C}(A)$ is strongly generated by the set $\mathcal{C}(A)_{\ll \lambda}$.

Proof. The inclusion $\mathcal{C}(A)[\lambda] \subset \mathcal{C}(A)$ is obvious. Since the cardinality of $A$ is smaller than the cofinality of $\lambda$, it is also clear that $A \in \mathcal{C}(A)[\lambda]$. Since $\mathcal{C}(A)[\lambda]$ is closed, we have $\mathcal{C}(A) \subset \mathcal{C}(A)[\lambda]$.

As a corollary we also get part 1 of Theorem 1.5.

4.7. Corollary. The order (Spaces, $\gg)$ is a complete lattice.

Proof. Let $\mathcal{J}$ be a set of spaces. For any choice of basepoints in the spaces of $\mathcal{J}$, $\inf (\mathcal{J})=\bigvee_{A \in \mathcal{J}} A$.

Note that $\sup (\mathcal{J})$ is represented by a cellular generator of $\bigcap_{A \in \mathcal{J}} \mathcal{C}(A)$. To show the existence of such a generator choose a limit ordinal $\lambda$ whose cofinality is bigger than the cardinalities of spaces in $\mathcal{J}, \# \mathcal{J}$, and $\mathbf{N}$. According to Proposition 4.4 and Corollary 4.6 we have equalities:

$$
\left(\bigcap_{A \in \mathcal{J}} \mathcal{C}(A)\right)[\lambda]=\bigcap_{A \in \mathcal{J}}(\mathcal{C}(A)[\lambda])=\bigcap_{A \in \mathcal{J}} \mathcal{C}(A)
$$

Since any class of the form $\mathcal{C}[\lambda]$ has a cellular generator (see Corollary 4.5), then so does $\bigcap_{A \in \mathcal{J}} \mathcal{C}(A)$.

To prove Propositions 4.3 and 4.4 we start with showing that, in the case $\mathcal{C}$ is closed, $\lambda$-C -small spaces are preserved by many operations. We would like to understand to what extent weak equivalences preserve $\lambda$-C $\mathcal{C}$-smallness.

4.8. Lemma. Let $\mathcal{C}$ be closed and $\lambda$ be a limit ordinal whose cofinality is bigger than $\mathbf{N}$ or $\lambda=\mathbf{N}$.

(1) Let $X \rightarrow Y \rightarrow X$ be maps whose composition is the identity. If $Y$ is $\lambda$-C-small, then so is $X$. 
(2) If $E \stackrel{\sim}{\rightarrow} B$ is an acyclic fibration, then $E$ is $\lambda$-C-small if and only if $B$ is.

(3) Let $I$ be an arbitrary set and $X=\operatorname{colim}\left(X_{0} \leftarrow \coprod_{I} \Delta[n, k] \hookrightarrow \coprod_{I} \Delta[n]\right)$. If $X_{0}$ is $\lambda$-C-small, then so is $X$.

(4) Let $X=\operatorname{colim}\left(X_{0} \hookleftarrow X_{1} \hookrightarrow X_{2}\right)$, where the indicated arrows are cofibrations. If, for all $i, X_{i}$ is $\lambda$-C-small, then so is $X$.

(5) Let $X=\operatorname{colim}\left(X_{0} \hookrightarrow X_{1} \hookrightarrow X_{2} \hookrightarrow \cdots\right)$, where the indicated arrows are cofibrations. If, for all $i, X_{i}$ is $\lambda$-C-small, then so is $X$.

(6) Let $I$ be an arbitrary set. If, for all $i \in I, X_{i}$ is $\lambda$-C-small, then, for any choice of basepoints in $X_{i}$, so is $\bigvee_{i \in I} X_{i}$.

(7) If $X$ is $\lambda$-C-small, then any map $X \rightarrow Y$ can be expressed as a composition $X \stackrel{\sim}{\hookrightarrow} X^{\prime} \rightarrow Y$, where $X^{\prime}$ is $\lambda$-C-small and the indicated maps are an acyclic cofibration and a fibration.

(8) Let $X \stackrel{\sim}{\rightarrow} Y$ be a weak equivalence. If $X$ is $\lambda$-C-small, then so is $Y$.

(9) Let $X \stackrel{\sim}{\rightarrow} Y$ be a weak equivalence. If $Y$ is $\lambda$-C -small and $X$ is fibrant, then $X$ is also $\lambda$-C-small.

(10) If $X \in \mathcal{C}[\lambda]$ and $X$ is fibrant, then $X$ is $\lambda$-C-small.

Proof of (1). This follows directly from the definitions.

Proof of (2). This part follows easily from the fact that any map $K \rightarrow B$ factors as $K \rightarrow E \stackrel{\sim}{\rightarrow} B$.

Proof of (3). Let $K \rightarrow X$ be a map where \#K is smaller than the cofinality of $\lambda$. Note that there is $J \subset I$ for which $K \rightarrow X$ factors through

$$
X^{\prime}=\operatorname{colim}\left(X_{0} \leftarrow \coprod_{J} \Delta[n, k] \hookrightarrow \coprod_{J} \Delta[n]\right)
$$

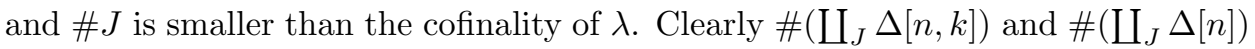
are also smaller than the cofinality of $\lambda$.

By pulling back $K \rightarrow X^{\prime}$ along the appropriate maps we can form the following commutative diagram where the squares on the right are pull-backs:

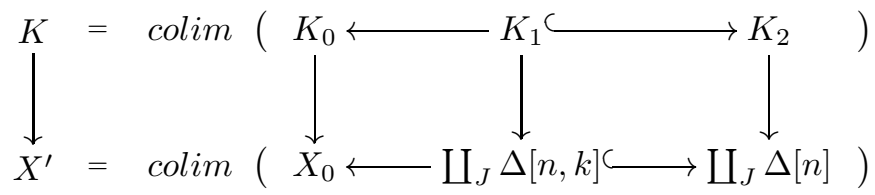

Since $\# K$ and $\# J$ are smaller than the cofinality of $\lambda$, the same is true for $\# K_{i}$, and thus also for the cardinality of $P=\operatorname{colim}\left(K_{0} \leftarrow K_{1} \rightarrow \coprod_{J} \Delta[n, k]\right)$. Let $P \rightarrow X_{0}$ be the map induced by the above diagram. By the assumption we can express $P \rightarrow X_{0}$ as $P \rightarrow Y \rightarrow X_{0}$, where $Y \in \mathcal{C}_{\ll \lambda}$. To get the desired factorization of $K \rightarrow X$, take $Y^{\prime}=\operatorname{colim}\left(Y \leftarrow \coprod_{J} \Delta[n, k] \hookrightarrow \coprod_{J} \Delta[n]\right)$. This space has cardinality smaller than the cofinality of $\lambda$ and is weakly equivalent to $Y$, thus belongs to $\mathcal{C}_{\ll \lambda}$.

Proof of 4, 5, and 6 . Since the proofs are analogous we show only part 4 . Let us point out however that part 5 requires the assumption that either $\lambda=\mathbf{N}$ or the cofinality of $\lambda$ is bigger than $\mathbf{N}$.

Let $K \rightarrow X$ be a map where $\# K$ is smaller than the cofinality of $\lambda$. By pulling back $K \rightarrow X$ along the appropriate maps, we can form the following commutative 
diagram, where the squares on the right are pull-backs and the indicated arrows are cofibrations:

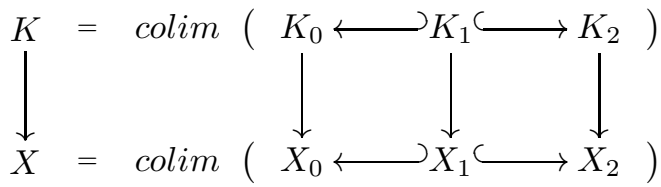

Since $K_{i} \subset K, \# K_{i}$ is smaller than the cofinality of $\lambda$. Let $K_{1} \rightarrow Y_{1} \rightarrow X_{1}$ be a factorization of $K_{1} \rightarrow X_{1}$ where $Y_{1} \in C_{\ll \lambda}$. Define $\overline{Y_{i}}=\operatorname{colim}\left(Y_{1} \leftarrow K_{1} \hookrightarrow K_{i}\right)$. There are natural maps $\overline{Y_{i}} \rightarrow X_{i}$. Since the cardinalities of $\overline{Y_{i}}$ are smaller than the cofinality of $\lambda$, we can find appropriate factorizations $\overline{Y_{i}} \hookrightarrow Y_{i} \rightarrow X_{i}$. The space $Y=\operatorname{colim}\left(Y_{0} \hookleftarrow Y_{1} \hookrightarrow Y_{2}\right)$ gives the desired factorization of $K \rightarrow X$.

Proof of (7). This follows from parts 3 and 5 and the standard way of factoring a map in Spaces into an acyclic cofibration followed by a fibration (the acyclic cofibrations are generated by the inclusions of horns into simplexes).

Proof of (8). According to part 7 we can factor $X \stackrel{\sim}{\rightarrow} Y$ as $X \stackrel{\sim}{\hookrightarrow} X^{\prime} \stackrel{\sim}{\rightarrow} Y$ where $X^{\prime}$ is $\lambda$-C-small. Part 8 follows now from part 2 .

Proof of (9). Factor $X \stackrel{\sim}{\rightarrow} Y$ by an acyclic cofibration followed by an acyclic fibration $X \stackrel{\sim}{\hookrightarrow} Y^{\prime} \stackrel{\sim}{\rightarrow} Y$. According to part $2, Y^{\prime}$ is $\lambda$-C -small. Since $X$ is fibrant, it is a retract of $Y^{\prime}$. Part 9 follows now from part 1 .

Proof of Proposition 4.3. In view of Lemma 4.8, to prove the proposition it is enough to show that $\mathcal{C}[\lambda]$ is closed under weak equivalences.

Let $X \stackrel{\sim}{\rightarrow} Y$ be a weak equivalence. We need to prove that $X \in \mathcal{C}[\lambda]$ if and only if $Y \in \mathcal{C}[\lambda]$. Assume that $X \in \mathcal{C}[\lambda]$. By possibly factoring a given map into a cofibration followed by an acyclic fibration, using part 2 of Lemma 4.8, we can assume that there is an acyclic cofibration $X \stackrel{\sim}{\hookrightarrow} X^{\prime}$, where $X^{\prime}$ is $\lambda$-C $\mathcal{C}$-small. Let $Y^{\prime}=\operatorname{colim}\left(X^{\prime} \stackrel{\sim}{\longleftarrow} X \stackrel{\sim}{\rightarrow} Y\right)$. Since $X^{\prime}$ is $\lambda$-C-Small, part 8 of Lemma 4.8 implies that so is $Y^{\prime}$. It follows that $Y \in \mathcal{C}[\lambda]$.

Assume that $Y \in \mathcal{C}[\lambda]$. Let $Y \stackrel{\sim}{\rightarrow} Y^{\prime}$ be a weak equivalence where $Y^{\prime}$ is $\lambda$ - $\mathcal{C}$-small. The composition $X \stackrel{\sim}{\rightarrow} Y \stackrel{\sim}{\rightarrow} Y^{\prime}$ gives the desired weak equivalence.

Proof of Proposition 4.4. The inclusion $\left(\bigcap_{i \in I} \mathcal{C}_{i}\right)[\lambda] \subset \bigcap_{i \in I}\left(\mathcal{C}_{i}[\lambda]\right)$ is clear since, for any $i \in I,\left(\bigcap_{i \in I} \mathcal{C}_{i}\right)[\lambda] \subset \mathcal{C}_{i}[\lambda]$.

Let $X \in \bigcap_{i \in I}\left(\mathcal{C}_{i}[\lambda]\right)$. This means that, for any $i \in I$, there is an acyclic cofibration $X \stackrel{\sim}{\hookrightarrow} X_{i}$ where $X_{i}$ is $\lambda-\mathcal{C}_{i}$-small. Define $Z:=\bigcup_{X} X_{i}$. Observe that the inclusions $X_{i} \hookrightarrow \bigcup_{X} X_{i}$ are weak equivalences. Part 8 of Lemma 4.8 implies that, for all $i \in I, Z$ is $\lambda-\mathcal{C}_{i}$-small. Since $X \rightarrow Z$ is also a weak equivalence, to show that $X \in\left(\bigcap_{i \in I} \mathcal{C}_{i}\right)[\lambda]$ it is enough to prove that $Z$ is $\lambda$ - $\left(\bigcap_{i \in I} \mathcal{C}_{i}\right)$-small.

Let $K \rightarrow Z$ be a map, where $\# K$ is smaller than the cofinality of $\lambda$. We need to prove that this map factors as $K \rightarrow Y \rightarrow Z$, where $\# Y$ is smaller than the cofinality of $\lambda$ and $Y \in \bigcap_{i \in I} \mathcal{C}_{i}$. We will construct $Y$ as the "telescope" of a diagram indexed by a certain ordinal number. Let us choose a well ordering on the set $I$ and denote by $e$ its smallest element. Define $\kappa$ to be the following order on $I \times \mathbf{N}$. Set $(i, m)<(j, n)$ if $m<n$ in $\mathbf{N}$ or $m=n$ and $i<j$ in $I$. Our strategy is to define, by induction, a diagram $F:[\kappa] \rightarrow$ Spaces together with a natural 
transformation $F \rightarrow Z$ such that:

- the map $K \rightarrow Z$ factors as $K \rightarrow F(e, 0) \rightarrow Z$;

- for any $(i, n) \in \kappa, \# F(i, n)$ is smaller than the cofinality of $\lambda$;

- for any $(i, n) \in \kappa, F(i, n) \in \mathcal{C}_{i}$.

Assume that we have such a functor. Observe that the imposed assumptions on $I$ imply that \#hocolim ${ }_{[\kappa]} F$ is smaller than the cofinality of $\lambda$. Moreover, for any $i \in I$, hocolim $_{[\kappa]} F \in C_{i}$ since hocolim $_{[\kappa]} F \simeq$ hocolim $_{n \in\{0,1, \ldots\}} F(i, n)$. This shows that $K \rightarrow$ hocolim $_{[\kappa]} F \rightarrow Z$ is the desired factorization.

Since $Z$ is $\lambda-C_{e}$-small we can find a factorization $K \rightarrow Y \rightarrow Z$, where $\# Y$ is smaller than the cofinality of $\lambda$ and $Y \in C_{e}$. Define $F(e, 0):=Y$ and $F(e, 0) \rightarrow Z$ to be the map $Y \rightarrow Z$.

Assume that we have defined $F$ for all $(i, m)<(j, n)$. Consider the map hocolim $_{[j, n]} F \rightarrow Z$. Since \#hocolim ${ }_{[j, n]} F$ is smaller than the cofinality of $\lambda$, we can factor this map as hocolim $_{[j, n]} F \rightarrow Y \rightarrow Z$, where $Y \in \mathcal{C}_{j}$ and $\# Y$ is smaller than the cofinality of $\lambda$. Define $F(j, n):=Y$. It is clear that $F$ satisfies the above requirements.

\section{Parameterized cellular spaces}

To prove part 2 of Theorem [1.5 we need to discuss parameterized families of cellular spaces. Let $f: E \rightarrow B$ be a map. For any simplex $\sigma: \Delta[n] \rightarrow B$, define $d f(\sigma):=\lim (\Delta[n] \stackrel{\sigma}{\rightarrow} B \stackrel{f}{\leftarrow} E)$.

5.1. Definition. Let $\mathcal{C}$ be a class of spaces. A map $f: E \rightarrow B$ is called a relative $\mathcal{C}$-complex if for any simplex $\sigma: \Delta[n] \rightarrow B, d f(\sigma) \in \mathcal{C}$.

Let $\mathcal{C}$ be a closed class. Since $\mathcal{C}$ consists of nonempty spaces, a relative $\mathcal{C}$-complex $f: E \rightarrow B$ is always an onto map.

A key property of closed classes is given by:

5.2. Theorem (4]). Let $\mathcal{C}$ be a closed class. Let $H, G: I \rightarrow$ Spaces be diagrams and $\Psi: H \rightarrow G$ be a natural transformation. If for any $i \in I, \Psi_{i}: H(i) \rightarrow G(i)$ is a relative $\mathcal{C}$-complex, then for any basepoint in hocolim ${ }_{I} G$, the homotopy fiber of hocolim $_{I} \Psi:$ hocolim $_{I} H \rightarrow$ hocolim $_{I} G$ is in $\mathcal{C}$.

The above implies:

5.3. Corollary. Let $\mathcal{C}$ be a closed class.

(1) If $f: E \rightarrow B$ is a relative $\mathcal{C}$-complex, then for any basepoint, the homotopy fiber of $f$ is in $\mathcal{C}$.

(2) Let $H, G: I \rightarrow$ Spaces be diagrams and $H \rightarrow G$ be a natural transformation. If for any $i \in I, H(i) \rightarrow G(i)$ is a relative $\mathcal{C}$-complex and hocolim ${ }_{I} G$ is contractible, then hocolim ${ }_{I} H \in \mathcal{C}$.

Relative complexes enjoy the following properties:

5.4. Proposition. Let $\mathcal{C}$ be a closed class and $\lambda$ be a limit ordinal.

(1) If $E \rightarrow B$ is a relative $\mathcal{C}_{\ll \lambda}$-complex, then, for any $h: X \rightarrow B$, so is the pull-back $h^{*} E \rightarrow X$. 
(2) Consider the following commutative diagram where the indicated arrows are cofibrations:

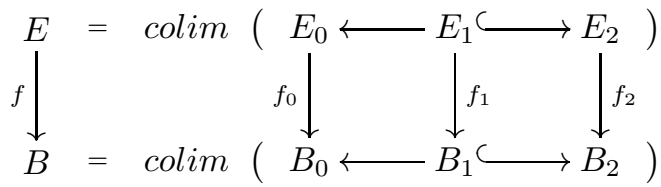

If $f_{i}$ are relative $\mathcal{C}_{\ll \lambda}$-complexes, then so is $f$.

(3) Let $\delta$ be an ordinal number smaller than the cofinality of $\lambda$. Let $\Psi: H \rightarrow G$ be a natural transformation between functors $H, G:[\delta] \rightarrow$ Spaces. Assume that for any $l<\delta$, colim $_{i<l} H(i) \hookrightarrow H(l)$ and $\operatorname{colim}_{i<l} G(i) \hookrightarrow G(l)$ are cofibrations and $\Psi_{l}: H_{l} \rightarrow G_{l}$ is a relative $\mathcal{C}_{\ll \lambda}$-complex. Then colim ${ }_{[\delta]} \Psi$ : $\operatorname{colim}_{[\delta]} H \rightarrow \operatorname{colim}_{[\delta]} G$ is also a relative $\mathcal{C}_{\ll \lambda}$-complex.

Proof. Part 1 is clear.

Since the proofs of parts 2 and 3 are analogous, we show only part 2 . We have to prove that $d f(\sigma) \in \mathcal{C}_{\ll \lambda}$, for any simplex $\sigma: \Delta[n] \rightarrow B$.

Denote by $K$ the image of the map $\sigma$. It is a finite subspace of $B$. Since the $f_{i}$ are relative $\mathcal{C}_{\ll \lambda}$-complexes, the subspace $f^{-1}(K) \subset E$ has cardinality smaller than the cofinality of $\lambda$. It follows that so does $d f(\sigma)$.

Let us consider the pull-backs:

$$
K_{i}=\lim \left(\Delta[n] \stackrel{\sigma}{\rightarrow} B \leftarrow B_{i}\right), \quad X_{i}=\lim \left(K_{i} \rightarrow B_{i} \stackrel{f_{i}}{\longleftarrow} E_{i}\right) .
$$

These spaces fit into the following commutative diagram where the indicated arrows are cofibrations:

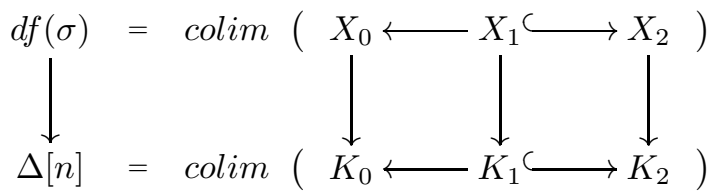

Since $f_{i}$ is a relative $\mathcal{C}$-complex, then so is $X_{i} \rightarrow K_{i}$, by part 1 . We can therefore use part 2 of Corollary 5.3 to conclude that $d f(\sigma)$ belongs to $\mathcal{C}$.

\section{Relatively "SMALL"-C-C-SPACES}

6.1. Theorem. Let $\lambda$ be a limit ordinal whose cofinality is bigger than $\mathbf{N}$ or $\lambda=\mathbf{N}$. If $\mathcal{C}$ is a closed class that is closed under extensions by fibrations, then so is $\mathcal{C}[\lambda]$.

As a corollary we get both the second part of Theorem 1.5 and Theorem 1.6.

6.2. Corollary. Let $A$ be a space. If the cofinality of $\lambda$ is bigger than \#A, then $\overline{\mathcal{C}(A)}=\overline{\mathcal{C}(A)}[\lambda]$. Moreover, $\overline{\mathcal{C}(A)}$ is strongly generated by the set $\overline{\mathcal{C}(A)}{ }_{\ll \lambda}$ and, for any choice of basepoints in the elements of $\overline{\mathcal{C}(A)}{ }_{\ll \lambda}, \overline{\mathcal{C}(A)}=\mathcal{C}\left(\bigvee_{X \in \overline{\mathcal{C}(A)}} X\right)$

Proof. The inclusion $\overline{\mathcal{C}(A)}[\lambda] \subset \overline{\mathcal{C}(A)}$ is clear. Since \#A is smaller than the cofinality of $\lambda, A \in \overline{\mathcal{C}(A)}[\lambda]$. Since $\overline{\mathcal{C}(A)}[\lambda]$ is a closed class that is closed under extensions by fibrations (see Theorem $[6.1), \overline{\mathcal{C}(A)} \subset \overline{\mathcal{C}(A)}[\lambda]$. The rest of the corollary follows from Corollary 4.5.

6.3. Corollary (Bousfield [2]). The order (Spaces, >) is a complete lattice. 
Proof. Let $\mathcal{J}$ be a set of spaces. For any choice of basepoints in the spaces of $\mathcal{J}$, $\inf (\mathcal{J})=\bigvee_{A \in \mathcal{J}} A$.

Note that $\sup (\mathcal{J})$ is represented by a cellular generator of the class $\bigcap_{A \in \mathcal{J}} \overline{\mathcal{C}(A)}$. Since, by Corollary 6.2, any class of the form $\overline{\mathcal{C}(A)}$ has a cellular generator, the existence of $\sup (\mathcal{J})$ follows from Corollary 4.7 .

To prove Theorem 6.1 we need to introduce a relative notion of $\lambda$-C -smallness.

6.4. Definition. Let $\mathcal{C}$ be a class of spaces and $\lambda$ be a limit ordinal. A map $p: X \rightarrow B$ is called $\lambda$-C -small if, for any $\alpha: K \rightarrow X$ where $\# K$ is smaller than the cofinality of $\lambda$, there exists a relative $\mathcal{C}_{\ll \lambda^{-}}$-complex $f: E \rightarrow B$ and a commutative diagram:

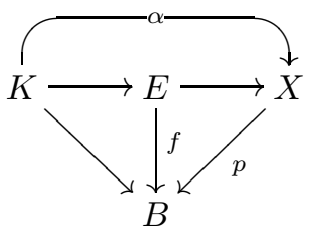

6.5. Remark. Observe that if $C$ is closed under weak equivalences, we can always arrange so that the map $K \rightarrow E$, in the above definition, is a cofibration. If not, let $E^{\prime}=\operatorname{colim}(E \leftarrow K \hookrightarrow K \times \Delta[1])$ and $E^{\prime} \rightarrow E$ be induced by $i d_{E}$ and $K \times \Delta[1] \stackrel{p r_{K}}{\rightarrow} K \rightarrow E$. By a direct verification one can show that the composition $E^{\prime} \rightarrow E \stackrel{f}{\rightarrow} B$ is also a relative $\mathcal{C}_{\ll \lambda^{-}}$complex

6.6. Lemma. Let $\mathcal{C}$ be a closed class and $\lambda$ be a limit ordinal whose cofinality is bigger than $\mathbf{N}$ or $\lambda=\mathbf{N}$. Let $p: X \rightarrow B$ be a fibration. If $\# B$ is smaller than the cofinality of $\lambda$ and, for any vertex $b \in B, p^{-1}(b)$ is $\lambda$-C-small, then $p$ is a $\lambda$-C -small map.

Proof. We first prove the lemma in the case when $B$ is contractible. Let $\alpha: K \rightarrow$ $X$ be a map, where $\# K$ is smaller than the cofinality of $\lambda$. Since in this case $p^{-1}(b) \hookrightarrow X$ is a weak equivalence, by part 8 of Lemma 4.8, $X$ is $\lambda$ - $\mathcal{C}$-small. We can find therefore a factorization $K \stackrel{g}{\rightarrow} Y \stackrel{h}{\rightarrow} X$ of $\alpha$ where $Y \in \mathcal{C}_{\ll \lambda}$. Consider now the following commutative diagram:

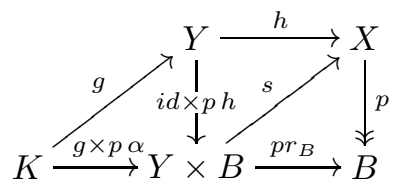

where $s$ is any lifting in the above square. Such a lifting does exists since $i d \times p h$ : $Y \rightarrow Y \times B$ is an acyclic cofibration. It is clear that $\operatorname{pr}_{B}: Y \times B \rightarrow B$ is the desired relative $\mathcal{C}_{\ll \lambda \text {-complex. }}$

For a general $B$, we prove the lemma by induction on the cell decomposition of $B$. Let $B=\operatorname{colim}\left(B_{0} \leftarrow \partial \Delta[n] \hookrightarrow \Delta[n]\right)$. Let $\alpha: K \rightarrow X$ be a map, where \#K is smaller than the cofinality of $\lambda$. By pulling-back $\alpha$ and $p$ along the appropriate maps we get the following commutative diagram where the squares on the right are 
pull-backs and the indicated arrows are respectively fibrations and cofibrations :

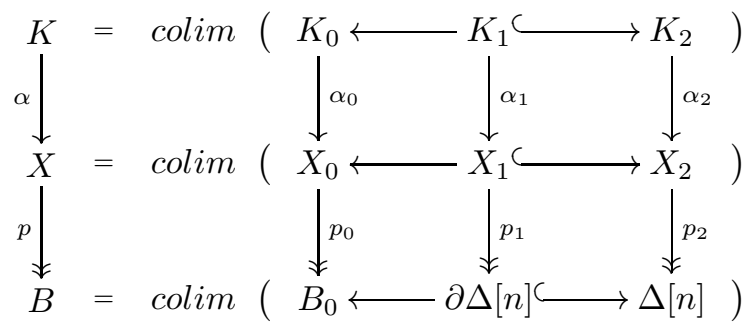

By the inductive assumption, the map $\alpha_{0}: K_{0} \rightarrow X_{0}$ yields an appropriate relative $\mathcal{C}_{\ll \lambda}$-complex $g_{0}: E_{0} \rightarrow B_{0}$. Define $E_{1}=\lim \left(E_{0} \stackrel{g_{0}}{\rightarrow} B_{0} \leftarrow \partial \Delta[n]\right)$. Let $g_{1}: E_{1} \rightarrow$ $\partial \Delta[n]$ be the induced map. According to part 1 of Proposition [5.4, $g_{1}$ is also a

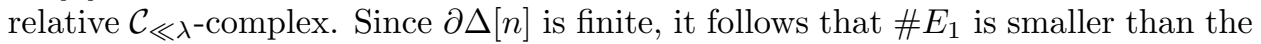
cofinality of $\lambda$. Let $P=\operatorname{colim}\left(E_{1} \leftarrow K_{1} \hookrightarrow K_{2}\right)$. It is clear that \#P is also smaller than the cofinality of $\lambda$. This space fits into a commutative diagram:

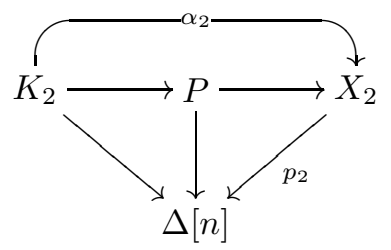

Since $\Delta[n]$ is contractible, $P \rightarrow X_{2}$ yields an appropriate relative $\mathcal{C}_{\ll \lambda^{-}}$-complex $g_{2}: E_{2} \rightarrow \Delta[n]$ where $P \hookrightarrow E_{2}$ is a cofibration (see Remark 6.5). Define $g: E \rightarrow B$ to be:

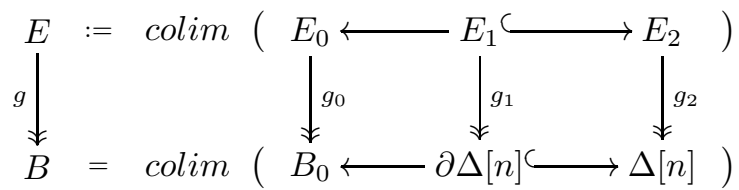

According to part 2 of Proposition 5.4 $g$ is a relative $\mathcal{C}_{\ll \lambda}$-complex. This concludes the lemma in the case when $B$ is finite. The case when $B$ is infinite follows from part 3 of Proposition 5.4.

Proof of Theorem [6.1 Let $p: X \rightarrow B$ be a map such that $B \in \mathcal{C}[\lambda]$ and, for any choice of a basepoint in $B, F i b(X \rightarrow B) \in \mathcal{C}[\lambda]$. We have to show that $X \in \mathcal{C}[\lambda]$.

Since $\mathcal{C}[\lambda]$ is closed under weak equivalences, we can arrange so that $p$ is a fibration and $B$ is fibrant. It follows from part 10 of Lemma 4.8 that spaces $B$ and $p^{-1}(b)$, for any vertex $b$ in $B$, are $\lambda$-C-small. To prove the theorem it is enough to show that $X$ is also $\lambda$-C-Small. Let $\alpha: K \rightarrow X$ be a map where $\# K$ is smaller than the cofinality of $\lambda$. We can factor the composition $p \alpha: K \rightarrow B$ into $K \rightarrow B^{\prime} \rightarrow B$, where $B^{\prime} \in \mathcal{C}_{\ll \lambda}$. Let us pull back $p$ along $B^{\prime} \rightarrow B$ and form the following commutative diagram:

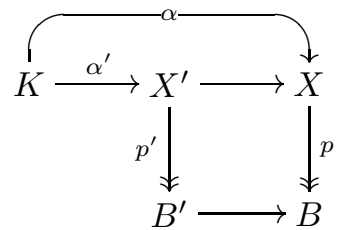


The fibration $p^{\prime}: X^{\prime} \rightarrow B^{\prime}$ satisfies the assumptions of Lemma 6.6. Let $g: E \rightarrow B^{\prime}$ be the induced relative $\mathcal{C}_{\lambda}$-complex for $\alpha^{\prime}: K \rightarrow X^{\prime}$. It is clear that $E \in \mathcal{C}$ since $\mathcal{C}$ is closed under extensions by fibrations. It is also clear that \#E is smaller than the cofinality of $\lambda$.

\section{REFERENCES}

[1] A. K. Bousfield, Localization and periodicity in unstable homotopy theory, J. Amer. Math. Soc. 7 (1994), no. 4, 831-873. MR 95c:55010

[2] — Homotopical localizations of spaces, Amer. J. Math. 119 (1997), no. 6, 1321-1354. MR 98m:55009]

[3] A. K. Bousfield and D. M. Kan, Homotopy limits, completions and localizations, Lecture Notes in Mathematics, Vol. 304, Springer-Verlag, Berlin, 1972. MR 51:1825

[4] Wojciech Chachólski, Closed classes, Algebraic topology: New trends in localization and periodicity (Sant Feliu de Guíxols, 1994), Birkhäuser, Basel, 1996, pp. 95-118. MR 97e:55007

[5] _ On the functors $C W_{A}$ and $P_{A}$, Duke Math. J. 84 (1996), no. 3, 599-631. MR 97i:55023

[6] Krzysztof Ciesielski, Set theory for the working mathematician, Cambridge University Press, Cambridge, 1997. MR 99c:04001

[7] Edward B. Curtis, Simplicial homotopy theory, Advances in Math. 6 (1971), 107-209 (1971). MR 43:5529

[8] E. Dror Farjoun, Cellular inequalities, The Čech centennial (Boston, MA, 1993), Amer. Math. Soc., Providence, RI, 1995, pp. 159-181. MR 96g:55011

[9] _ Cellular spaces, null spaces and homotopy localization, Springer-Verlag, Berlin, 1996. MR 98f:55010

[10] Daniel G. Quillen, Homotopical algebra, Lecture Notes in Mathematics, No. 43, SpringerVerlag, Berlin, 1967. MR 36:6480

Yale University, Department of Mathematics, 10 Hillhouse Avenue, P.O. Box 208283, New Haven, Connecticut 06520-8283

E-mail address: chachols@math.yale.edu

Current address: KTH Matematik, S-10044 Stockholm, Sweden

Université Catholique de Louvain, Département de mathméatiques, 2 Chemin du CyClotron, B-1348 Louvain-la-Neuve, Belgique

E-mail address: parent@agel.ucl.ac.be

Current address: KTH Matematik, S-10044 Stockholm, Sweden

University of Alberta, Department of Mathematical Sciences, 632 Central Academic Building, Edmonton, Alberta, T6G 2G1, Canada

E-mail address: stanley@math.ualberta.ca

Current address: Department of Mathematics and Statistics, University of Regina, College West, 30714 Regina, Saskatchewan, Canada 